\title{
Efectividad de los productos empleados en la prevención de la radiodermatitis en pacientes con cáncer de mama. Revisión Sistemática
}

FOLCH-AYORA A ${ }^{\mathrm{a}}$ VINUESA-BARCELO A ${ }^{\mathrm{a}}$; SUAREZ-ALCAZAR MP ${ }^{\mathrm{b}}$, SALASMEDINA $P^{\mathrm{a}}$, COLLADO-BOIRA E ${ }^{\mathrm{a}}$, BERNAT-ADELL MD ${ }^{\mathrm{a}}$

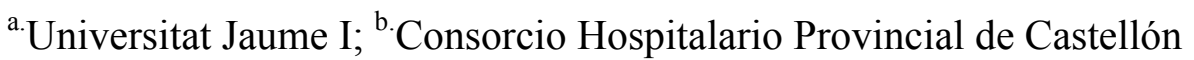

Arantxa Vinuesa Barceló RN

Ana Folch Ayora RN, MSc, PhD

María Pilar Suarez Alcazar RN

Pablo Salas Medina RN, MSc, PhD

Corresponding autor: Ana Folch Ayora, Profesora Unidad Predepartamental de Enfermería, Facultad de Cièncias de la Salud, Universitat Jaume I, Castellón, Av. de Vicent Sos Baynat, s/n , 12071 Castellón de la Plana (Spain); Phone: +34 964387745

FINANCIACIÓN: Este estudio ha sido parcialmente subvencionado por la Universitat Jaume I (UJI-A2017-06)

CONFLICTO DE INTERESES: Los autores declaran que no existe ningún conflicto de intereses en la publicación de este estudio. 


\section{RESUMEN}

Introducción. La radioterapia es uno de los tratamientos más utilizados en los pacientes paliativos siendo la radiodermatitis el efecto secundario más frecuente de la radioterapia, afectando a un $85-95 \%$ de los pacientes con cáncer sometidos a este tratamiento. Objetivo: El propósito de esta revisión es identificar aquellos productos con mayor eficacia en la prevención de la radiodermatitis en los pacientes con cáncer de mama.

Métodología. Revisión sistemática de ensayos clínicos aleatorizados sobre la prevención de la radiodermatitis en pacientes con cáncer de mama en tratamiento radioterápico no concomitante, recopilados en las bases de datos PubMed, Lilacs, CINHAL, Trip Databases y Proquest Health \& Medical Complete, publicados en los últimos 6 años (2021 a 2018) en lengua inglesa y española.

Resultados. Los productos que fueron efectivos en la prevención de la radiodermatis son: la glutamina enteral, la curcumina oral, los apósitos de film transparente, la sulfadiazina de plata, la película o la crema de barrera con polimeros, el furoato de mometasona, la crema con hidrocortisona al $1 \%$, la crema con melatonina y la crema con factor de crecimiento epidérmico (EGF). Se desaconseja el uso del ácido hialurónico y del aloe vera. No mejoró la radiodermatitis con el empleo de cremas hidratantes, caléndula, boswellia, alantoína y el aceite de emú.

Conclusiones. En la prevención de la dermatitis por radioterapia, es efectivo el empleo de productos cuya finalidad sea la protección de la piel, como los apósitos tipo film y las cremas o películas con barrera de polimeros. Además de productos potenciadores del sistema inmunitario (glutamina enteral). Y productos con propiedades antioxidantes, (curcumila oral, la crema de melatonina o el factor de crecimiento epidérmico). Otros productos como los corticoides y los antibióticos tópicos, son efectivos pero su uso a largo plaza conlleva efectos secundarios.

Palabras clave: Prevención, Radiodermatitis, Neoplasias de la mama, Radioterapia. 


\section{Introducción}

La radioterapia es uno de los pilares terapéuticos del cáncer y especialmente en los paciente con problemas asociados a la evolución de su enfermedad, causando su uso diversos efectos secundarios, siendo los más frecuentes los relacionados con la piel debido a la alta radiosensibilidad y susceptibilidad a ser dañada por las radiaciones (1).

Desde la primera dosis de radiación hasta la última se produce un daño continuado en las células epiteliales tanto de origen benigno como maligno, ya que el mecanismo de acción empleado por la radioterapia desencadena la destrucción tisular continua y evita la reparación del tejido dañado(1). Los efectos secundarios comienzan a ser evidentes desde la segunda o tercera semana de tratamiento(2).

Así pues, las cifras de radiodermatitis desarrollada en los pacientes con tratamiento radioterápico se sitúan entre el 85\% y el 90\%(3). Su presentación se basa en la presencia de reacciones cutáneas físicas que generan malestar como el picazón, el dolor, la incomodidad, la irritación y el ardor(1). Que pueden variar según The Radiation Therapy Oncology Group Criteria, desde: Grado 1 (GI) eritema leve a dosis de 2Gys, Grado 2 (GII) descamación seca a dosis de 2-4 Gys, Grado 3 (GIII) descamación húmeda a dosis de 4Gys y Grado 4 (GIV) necrosis(1).

La gravedad de la afectación cutánea depende de múltiples factores, algunos relacionados con el paciente, como la edad, el índice de masa corporal, el consumo de tabaco y alcohol, el tamaño del tumor, el color de la piel, las comorbilidades (diabetes) y otros relacionados con el tratamiento, como el tipo de haz, la cantidad de energía empleada, el volumen de tratamiento, la dosis total, el fraccionamiento o la asociación con quimioterapia $(4,5)$.

Los cambios en la piel por radiación pueden afectar las actividades de la vida diaria y la calidad de vida(6). Aunque la presencia de radiodermatitis no supone un riesgo para la vida de las personas pese a producir una mayor sensibilidad a los alérgenos, las radiaciones ultravioleta y a las infecciones(2); puede volverse incómoda si se realiza un mal manejo(7) pudiendo ser necesaria la interrupción del tratamiento(8).

Conscientes de dicho problema se han llevado a cabo numerosas investigaciones que evalúan las recomendaciones en el cuidado de la piel y los agentes tópicos que puedan prevenir y tratar la radiodermatitis(9). Existiendo gran variedad de recomendaciones 
sobre el manejo y el uso de productos $(3,8)$. Dependiendo en muchas ocasiones solamente de la experiencia clínica o de la disponibilidad de determinados agentes tópicos $(8,10)$.

La Guía de Práctica Clínica publicada por la Oncology Nursing Society(10) evidencia la eficacia durante la radioterapia de: 1) medidas higiénicas (uso de jabones con $\mathrm{pH}$ neutro, desodorante y secado suave), y 2) recomendaciones de seguridad (evitar la exposición solar o usar ropa sin fibras sintéticas). Así pues La Guía de Práctica Clínica publicada por la Oncology Nursing Society(10) concluye que no se pueden establecer recomendaciones sobre productos que prevengan la aparición de la radiodermatitis, debido a la gran diversidad y heterogeneidad de productos tópicos empleados en el control de las reacciones cutáneas(11).

Por tanto, objetivo de este trabajo es realizar una revisión sistemática de la evidencia disponible en base a los ensayos clínicos publicados que evaluaran la efectividad de los tratamientos tópicos y orales en pacientes con cáncer de mama tratados con radioterapia externa. Con la finalidad de poder dar una información y unos cuidados preventivos actualizados.

\section{Metodología}

\section{$\underline{2.1 \text { Diseño }}$}

Revisión sistemática de los Ensayos Clínicos publicados en los últimos seis años (2012 2018) sobre la efectividad de los productos tópicos empleados en la prevención de la radiodermatitis en pacientes con diagnóstico de cáncer de mama sometidos a radioterapia.

\subsection{Pregunta}

La pregunta de investigación de este estudio fue: ¿Qué productos de uso tópico son efectivos para la prevención de la radiodermatitis desarrollada por el tratamiento radioterápico en pacientes con diagnóstico de cáncer de mama?

\section{$\underline{2.3 \text { Estrategia de búsqueda }}$}

Para la búsqueda de la literatura se emplearon los descriptores del Medical Subject Headings (MeSH) "radiodermatitis" y "breast neoplasms" usando el operador booleano "AND" entre ambos en las bases de datos PubMed, Lilacs, CINHAL, Trip Databases y 
Proquest Health \& Medical Complete. Para la delimitación de esta búsqueda se emplearon varios filtros: límites cronológicos (artículos publicados en los últimos seis años, desde enero de 2012 hasta enero de 2018), idiomáticos (lengua española e inglesa), de especie (en humanos) y en cuanto a la tipología de estudio, se seleccionaron sólo ensayos clínicos aleatorizados.

\section{$\underline{2.4 \text { Criterios de selección }}$}

Criterios de inclusión: Pacientes mayores de 18 años con diagnóstico primario de cáncer de mama confirmado histológicamente independientemente del estadio, que vayan a recibir tratamiento de radioterapia, no concomitante y que no tengan radiodermatitis previa a la inclusión en el estudio. No se tuvieron en cuenta aspectos como el sexo, el país de procedencia o la etnia.

Criterios de exclusión: Estudios que utilicen la modificación de la dosis de radiación a recibir como tratamiento preventivo. También se excluyeron los protocolos de actuación, revisiones sistemáticas o guías de práctica clínica enfermera.

\section{$\underline{2.5 \text { Selección de los estudios }}$}

Los pasos establecidos en esta investigación fueron: establecimiento de la pregunta de investigación, establecimiento de los criterios de inclusión y exclusión, búsqueda bibliográfica e inspección de los títulos y resúmenes de las referencias identificadas en la búsqueda para evaluar la cumplimentación de los criterios de inclusión y exclusión. La elección de los Ensayos Clínicos y la lectura crítica de los textos completos se llevó a cabo por dos revisores (enfermeras) de forma independiente y cegada. En caso de discrepancias se solicitó la evaluación de una tercera persona. Para la recogida de datos se elaboraron unas tablas que contenían las variables a estudio, para facilitar la categorización y la síntesis de los resultados.

Para la evaluación de la calidad metodológica de los estudios se utilizó la Guía de Valoración crítica de Ensayos Clínicos Aleatorizados del Critical Appraisal Skill Program en español (CASPe). Dicha escala consta de 11 ítems, siendo los 3 primeros de exclusión y los 7 siguientes complementarios. Así pues, se atribuyeron dos criterios de exclusión: el primero, el no cumplimiento de los 3 primeros ítems, y el segundo, la no obtención de una puntuación mayor o igual a 6 sobre 11 . 


\section{$\underline{2.6 \text { Variables del estudio }}$}

- Calidad metodológica: Puntuación en la escala CASPe.

- Características de la población a estudio: participantes iniciales, participantes finales, \% de pérdidas, periodo de evaluación expresado en meses, sexo, edad, tipo de tumor y estadio tumoral.

- Características del tratamiento radioterápico: Grays (Gy) totales, Gy por sesión, escala de valoración de la radiodermatitis por radioterapia y grado de radiodermatitis desarrollado.

- Productos empleados: productos, ver tabla 1, vía de administración, producto administrado en el Grupo Intervención (GI), productor empleado en el Grupo Control (GC), Composición del producto del GC y grado de significación de la efectividad entre los productos del GI y el GC.

\subsection{Análisis de los datos}

Tras categorización de las variables objeto de estudio se realizó una síntesis cuantitativa, mediante la media (x), y cualitativa, mediante las frecuencias y los porcentajes (n, \%).

Debido a la heterogeneidad de los estudios se consideró adecuado que los resultados obtenidos se sintetizaran mediante un resumen de los p-value obtenidos, para poder dar respuesta a la pregunta de búsqueda.

\section{Resultados}

\section{$\underline{3.1 \text { Resultados del proceso }}$}

Tras aplicar la estrategia de búsqueda se localizaron 86 artículos, de los cuales 19 estaban duplicados, tras lectura de título y resumen fueron eliminados 47 , quedando un total de 20 artículos leídos a texto completo, descartando 2 por ser protocolos de investigación y no presentar resultados. Por tanto un total de 18(4,5,12-27) artículos fueron seleccionados para la síntesis cualitativa y cuantitativa de esta revisión sistemática. Ver Figura 1.

\section{$\underline{3.2 \text { Calidad metodológica }}$}

La puntuación media del CASPe se situó en 8,1 puntos, existiendo resultados que variaron de $6(\mathrm{n}=1)(26)$ a $9(\mathrm{n}=8)(4,5,13,14,19,20,22,25)$ puntos, no descartándose ningún artículo por puntuaciones inferiores a 6 , ver Tabla 2 . 


\section{$\underline{3.3 \text { Resultados de los estudios }}$}

El número total de participantes fue de 2.324, de los cuales, 2.188 finalizaron el estudio. Con una media de 122 participantes y una tasa media de abandono del 5\%. Estando descrito el periodo de evaluación en el 72\% $(n=13)(4,12-19,24-27)$ de los estudios, con periodos que van desde los 6(4) hasta los 28(27) meses, con una media de duración de 15 meses por estudio, ver Tabla 2.

Si atendemos a las variables sociodemográficas en el $89 \%(n=16)(4,5,13-25,27)$ de los estudios la población fue exclusivamente femenina y en un $11 \%(n=2)(12,26)$ mixta. La edad media varío entre 48,1(19) y 60,4(26) años, situándose la media en 55,5 años, ver Tabla 2.

El 100\% $(\mathrm{n}=18)(4,5,12-27)$ de los estudios están realizados en pacientes con diagnóstico de cáncer de mama, siendo un 94\% $(n=17)(4,5,12-25,27)$ exclusivos de esta tipología de tumor y un $6 \%(\mathrm{n}=1)(26)$ sobre cáncer de mama, pulmón y cabeza y cuello. Si atendemos al estadio tumoral en el $66.7 \%(n=12)(15-18,20-27)$ no se hizo referencia a dicho aspectos, donde en aquellos estudios que si lo reflejaron, el 33.3\% $(n=6)(4,5,12-14,18)$ incluyeron participantes con un estadío tumoral del 0 al IV, ver Tabla 2.

\section{$\underline{3.4 \text { Resultados relacionados con el tratamiento radioterápico }}$}

En el 39\% $(n=7)(4,12,13,16,17,20,23)$ de los estudios se emplearon para el tratamiento radioterápico energía de fotones, en el $11 \%(n=2)(15,19)$ de electrones, y en un $11 \%$ $(\mathrm{n}=2)(18,24)$ ambos. Un 6\% $(\mathrm{n}=1)(5)$ emplearon la energía de fotones de cobalto $60 \mathrm{y}$ en el resto de estudios, un 33\% $(n=6)(14,21,22,25-27)$, no se hizo referencia al tipo de energía empleada, ver Tabla 3.

La dosis de radiación total en los estudios varío entre $20 \mathrm{~Gy}(22)$ y $50 \mathrm{~Gy}(4,15,16,18-$ 20,26), obteniendo una dosis de radiación media de 44,61Gy. En cuanto a la dosis de radiación por sesión, osciló entre $1,75 \mathrm{~Gy}(21)$ y 2,67Gy(24), con una radiación media por estudio de 1,97Gy por sesión, ver Tabla 3.

\section{$\underline{3.5 \text { Resultados prevención de la radiodermatitis }}$}

Las escalas empleadas para la valoración de la radiodermatitis fueron la RTOG, en un $50 \%(n=9)(12,13,16,17,19,20,22,24,25)$ la CTCAE en un $33 \%(n=6)(5,15,18,21,26,27)$, ambas en un $6 \%(\mathrm{n}=1)(4)$ y en un $11,1 \%(\mathrm{n}=2)$ la $\operatorname{CSSP}(23)$ y la $\operatorname{RDS}(14)$, ver Tabla 4. 
En el 89\% $(\mathrm{n}=16)(4,5,12,13,15-21,23-27)$ la vía de administración del tratamiento para la prevención de la radiodermatitis fueron los productos tópicos como los: aceites(21), cremas(4,5,13,15-17,19,20,23,24,27), geles(18), emulsiones(26) y apósitos (12), para la prevención de la radiodermatitis y el $11 \%(n=2)(14,22)$ restante productos orales, ver Tabla 4.

En el $17 \%(n=3)(4,5,20)$ de los estudios en el grupo control se administró un placebo. Y en el $61 \%(n=11)$ se usó una crema hidratante $(n=4)(16,23,24,27)$, crema acuosa $(n=3)(12,17,26)$, aceite de semilla de algodón $(n=1)(21)$, gel de vaselina $(n=1)(18)$, glucosa oral $(n=1)(22)$ y cápsulas con colorante $(n=1)(14) ; \quad y$ en el $22 \%$ $(\mathrm{n}=4)(13,15,19,25)$ restante, no se dio ningún producto, ver Tabla 4.

Los productos tópicos que fueron efectivos con un $\mathrm{p}<0.05$ en la prevención de la radiodermatitis fueron: crema con melatonina(4), crema de hidrocortisona $1 \%(5)$, furoato de mometasona $0,1 \%(24)$, crema de barrera de polimeros(27), crema de EGF(13). Y en un $p<0.01$ fueron efectivos: el apósito de film transparente(12), la película de barrera con polimeros(15) y la crema de sulfadiazina de Plata(19). En cuanto a los productos administrados por vía oral fueron efectivos en un $p<0.01$, la glutamina enteral(22) y la curcumina oral(14), ver tabla 4.

Otros productos como el aceite de emú(21), la crema hidratante(16), la emulsión con alantoína(26) y la crema de caléndula(17), no obtuvieron resultados estadísticamente significativos en la prevención de la aparición de radiodermatitis. Sin embargo, la crema de aloe vera(23) y el gel de ácido hialurónico(18) precipitaron la aparición de radiodermatitis, frente al grupo placebo $(\mathrm{p}<0.05)$, ver Tabla 4.

Se desaconseja el uso del ácido hialurónico y la aloe vera. No mejoró la radiodermatitis con el empleo de cremas hidratantes, caléndula, boswellia, alantoína y el aceite de emú.

\section{Discusión}

Un total de 18 ensayos clínicos han estudiado el efecto de la melatonina, el aceite de emú, la glutamina, las barreras protectoras con polímeros tópicos, la hidrocortisona, la broswellia, el aloe vera, el furorato de mometasona, la emulsión de alantoína, la cúrcuma, el ácido hialurónico, la sulfadiazina de plata, el factor epitelial de crecimiento epidérmico 
y la caléndula en la prevención de la dermatitis por radioterapia en pacientes con cáncer de mama, en los últimos 6 años.

El perfil de pacientes incluidos en estos estudios fueron mujeres con una edad media de 55,5 años. Datos que se corresponden con el perfil de pacientes diagnosticadas de cáncer de mama a nivel mundial(28).

En base a los resultados obtenidos, uno de los aspectos que condiciona la aparición de radiodermatitis, es la dosis de radiación administrada. Donde oscilaron entre 44,61Gy en total del tratamiento y $1,97 \mathrm{~Gy}$ por sesión. Datos que se corresponden con las pautas de tratamiento de las principales guías de abordaje terapéutico del cáncer de mama. Cuyos valores recomendados varían entre los 45Gy y 50 Gy en total y 2 Gy por sesión de radioterapia(29). Para la valoración y cuantificación de la radiodermatitis, la escala más empleada fue la CTCAE, desarrollada por el NCI en el año 1983(30).

Las vías de administración más utilizadas para la prevención de la radiodermatitis, fueron la vía tópica y la oral. Sobre esta última, destacó la efectividad de la glutamina enteral(22), con propiedades regenerativas y potenciadoras del sistema inmunitario y la curcumina oral(14), con potencial antiinflamatorio y antioxidantes. Su presencia ayuda a reconstruir los daños producidos en las células de los tejidos tratados con radioterapia(31). Cuyos beneficios también, han sido observados en la prevención y curación de quemaduras en pacientes ingresados en unidades de cuidados críticos(32).

En cuanto a los productos tópicos, tal y como han mostrado nuestros resultados, existe una gran heterogeneidad de presentaciones tópicas (aceites, cremas, geles, emulsiones, películas protectoras y apósitos). Donde no existe una presentación que resulte más efectiva, que otra. Este aspecto también quedo manifestado en el trabajo de Gosselin(33), basado en recomendaciones para el tratamiento de la radiodermatitis instaurada en pacientes con cáncer de mama.

En base a nuestros resultados, los productos más efectivos en la prevención de la radiodermatitis fueron los apósitos de film transparente(12). Dispositivos constituidos por un dispositivo de polímero fino transparente y transpirable, diseñado para la protección de la piel frágil y sensible con el objetivo de disminuir el riesgo de laceraciones(34). Mecanismo similar al empleado por las películas(15) o cremas(27) de barrera con polimeros, cuyos resultados han sido efectivos en la prevención de la radiodermatitis. 
Según estos resultados, los dispositivos de barrera, serían recomendables en la prevención de la radiodermatitis, por la protección primaria, que ofrecen frente a posibles lesiones. Otros productos, con mecanismo similares, como los apósitos de espuma han sido eficaces en el tratamiento de la radiodermatitis en pacientes con cáncer de mama(35,36). Pese a que no hemos encontrado evidencia, que trate sobre su efectividad en la prevención de la radiodermatitis.

Los tratamientos farmacológicos empleados por vía tópica fueron los corticoides como el furoato de mometasona(24) y la crema con hidrocortisona al 1\%(5). Obteniendo una reducción de la aparición de la radiodermatitis. Sin embargo, no todos los investigadores recomiendan su empleo, como consecuencia de la atenuación del sistema inmunitario(3841). Otro fármaco con efectos similares, es la sulfadiazina de plata, que reducen la aparición de radiodermatitis. Beneficios solo observados en el estudio de Hemati, et al.(19). Las ultimas recomendacines del Consensus on Wound Antisepsis, desaconsejan su aplicación por los mismo motivos que los corticoides tópicos(41).

Otros productos como la crema de melatonina(4) o el Factor de Crecimiento Epidérmico (EGF)(13), han sido eficaces. Donde en ambos casos el mecanismo de acción se basa en la reducción de los radicales libres de las células dañadas durante la radioterapia, facilitando la regeneración celular y reparando el daño tisular. Sin embargo, otros productos administrados por vía tópica como el ácido hialurónico(18), al que también se le atribuyen propiedades antioxidantes, ha mostrado resultados contraproducentes, facilitando la aparición de radiodermatitis. Resultados similares, son los obtenido con la aplicación de la aloe vera(23), cuyo mecanismo de acción, basado en la hidratación celular, podría actuar como factor potenciador del efecto de la radioterapia, situación que explicaría el efecto potenciador de la aparición de radiodermatitis. Así pues, tanto el ácido hialuronico como la aloe vera son utilizan de forma habitual en el tratamiento de la radiodermatitis instaurada, con excelentes resultados(42-45).

En cuanto al empleo de desodorantes, su uso esta desaconsejado en base a las guías de práctica clínica(46). Sin embargo, en base a los datos obtenidos, no se evidencia que respalde la mayor predisposición de desarrollar radiodermatitis tras el empleo de estos(25). Datos también corroborados por otros investigadores(47-49).

Otras recomendaciones empleados no localizados en esta revisión pero si descritos en la literatura son la urea al 3\% (50) aplicada de forma intensiva durante 3 veces/día dos 
semanas antes del tratamiento, o la crema de silimarina al $0,25 \%(51)$, han obtenido resultados satisfactorios en la prevención de la radiodermatitis

\section{Conclusión}

En la prevención de la dermatitis por radioterapia, es efectivo el empleo de productos cuya finalidad sea la protección de la piel, como los apósitos tipo film y las cremas o películas con barrera de polimeros. Además de productos potenciadores del sistema inmunitario como la glutamina enteral. Y productos con propiedades antioxidantes, que reduzcan los radicales libres de las células dañadas durante la radioterapia, como la curcumila oral, la crema de melatonina o el factor de crecimiento epidérmico. Otros productos como los corticoides (hidrocortisona $1 \%$, furoato de mometasona $0,1 \%$ ) y los antibióticos tópicos (sulfadiazina de plata), son efectivos pero su uso a largo plaza conlleva efectos secundarios. Productos la crema de boswellia $\%$, el aceite de emú, los desodorantes, la emulsión con alantoína, las cremas hidratantes y la crema con caléndula, no aportan beneficios ni perjuicios. Además, el uso del ácido hialuronico y la aloe vera estarían desaconsejados para la prevención de la dermatitis por radioterapia.

Esta investigación puede ser el inició de otras investigaciones que analicen, de forma conjunta, la efectividad de aquellos productos que apuntan a una reducción de la radiodermatitis, para poder establecer conclusiones más precisas.

\section{Bibliografía}

1. McQuestion M. Evidence-Based Skin Care Management in Radiation Therapy: Clinical Update. Semin Oncol Nurs [Internet]. 2011 May 1 [cited 2018 May 10];27(2):e1-17. Available from: https://www.sciencedirect.com/science/article/pii/S074920811100012X

2. Berger A, Regueiro C, Hijal T, Pasquier D, De La Fuente C, Le Tinier F, et al. Interest of Supportive and Barrier Protective Skin Care Products in the Daily Prevention and Treatment of Cutaneous Toxicity During Radiotherapy for Breast Cancer. Breast Cancer Basic Clin Res [Internet]. 2018 Jan 23 [cited 2018 May 10];12:117822341775277. Available from: http://journals.sagepub.com/doi/10.1177/1178223417752772

3. Fernández-Castro M, Martín-Gil B. Efectividad del tratamiento tópico en pacientes con cáncer de mama que sufren radiodermatitis. Una revisión sistemática. Enfermería Clínica [Internet]. 2015 Nov [cited 2018 May 10];25(6):327-43. Available from:

http://linkinghub.elsevier.com/retrieve/pii/S1130862115000820

4. Ben-David MA, Elkayam R, Gelernter I, Pfeffer RM. Melatonin for Prevention of Breast Radiation Dermatitis: A Phase II, Prospective, Double-Blind 
Randomized Trial. Isr Med Assoc J. 2016;18(3-4):188-92.

5. Meghrajani CF, Co HS, Arcillas JG, Maaño CC, Cupino NA. A randomized, double-blind trial on the use of $1 \%$ hydrocortisone cream for the prevention of acute radiation dermatitis. Expert Rev Clin Pharmacol. 2016 Mar;9(3):483-91.

6. De Conno F, Ventafridda V, Saita L. Skin problems in advanced and terminal cancer patients. J Pain Symptom Manage [Internet]. 1991 May 1 [cited 2018 May 10];6(4):247-56. Available from:

https://www.sciencedirect.com/science/article/pii/088539249190015V

7. Titeca G, Poot F, Cassart D, Defays B, Pirard D, Comas M, et al. Impact of cosmetic care on quality of life in breast cancer patients during chemotherapy and radiotherapy: an initial randomized controlled study. J Eur Acad Dermatology Venereol [Internet]. 2007 Jul [cited 2018 May 10];21(6):771-6. Available from: http://www.ncbi.nlm.nih.gov/pubmed/17567306

8. D 'haese S, Roy M Van, Bate T, Bijdekerke P, Vinh-Hung V. Management of skin reactions during radiotherapy in Flanders (Belgium): A study of nursing practice before and after the introduction of a skin care protocol. Eur J Oncol Nurs [Internet]. 2010 [cited 2018 May 10];14:367-72. Available from: https://ac.els-cdn.com/S1462388909001197/1-s2.0-S1462388909001197main.pdf? tid=264fd93e-532e-4fb2-9b56b1a0c8504d6b\&acdnat $=1525946200 \_0 f b b 9 f 4 d d 7 c f 60 e e 167 f 4 a a 8 b 23 f 0 a 52$

9. Kumar S, Juresic E, Barton M, Shafiq J. Management of skin toxicity during radiation therapy: A review of the evidence. J Med Imaging Radiat Oncol [Internet]. 2010 Jun 9 [cited 2018 May 10];54(3):264-79. Available from: http://doi.wiley.com/10.1111/j.1754-9485.2010.02170.x

10. Bolderston A, Lloyd NS, Wong RKS, Holden L, Robb-Blenderman L. The prevention and management of acute skin reactions related to radiation therapy: a systematic review and practice guideline. Support Care Cancer [Internet]. 2006 Aug 7 [cited 2018 May 10];14(8):802-17. Available from: http://link.springer.com/10.1007/s00520-006-0063-4

11. Menêses AG de, Reis PED dos, Guerra ENS, Canto GDL, Ferreira EB, Menêses AG de, et al. Use of trolamine to prevent and treat acute radiation dermatitis: a systematic review and meta-analysis. Rev Lat Am Enfermagem [Internet]. 2018 May 7 [cited 2018 May 10];26(0). Available from: http://www.scielo.br/scielo.php?script=sci_arttext\&pid=S0104$11692018000100600 \& \operatorname{lng}=\mathrm{en} \& \operatorname{tlng}=\mathrm{en}$

12. Herst PM, Bennett NC, Sutherland AE, Peszynski RI, Paterson DB, Jasperse ML. Prophylactic use of Mepitel Film prevents radiation-induced moist desquamation in an intra-patient randomised controlled clinical trial of 78 breast cancer patients. Radiother Oncol. 2014 Jan;110(1):137-43.

13. Kong M, Hong SE. Topical use of recombinant human epidermal growth factor (EGF)-based cream to prevent radiation dermatitis in breast cancer patients: a single-blind randomized preliminary study. Asian Pac J Cancer Prev. 2013;14(8):4859-64.

14. Ryan JL, Heckler CE, Ling M, Katz A, Williams JP, Pentland AP, et al. Curcumin for Radiation Dermatitis: A Randomized, Double-Blind, Placebo- 
Controlled Clinical Trial of Thirty Breast Cancer Patients. Radiat Res. 2013 Jul;180(1):34-43.

15. Shaw S-Z, Nien H-H, Wu C-J, Lui LT, Su J-F, Lang C-H. 3M Cavilon No-Sting Barrier Film or topical corticosteroid (mometasone furoate) for protection against radiation dermatitis: A clinical trial. J Formos Med Assoc. 2015 May;114(5):407-14.

16. Di Franco R, Sammarco E, Calvanese MG, De Natale F, Falivene S, Di Lecce A, et al. Preventing the acute skin side effects in patients treated with radiotherapy for breast cancer: the use of corneometry in order to evaluate the protective effect of moisturizing creams. Radiat Oncol [Internet]. 2013 Mar 12 [cited 2017 Mar 8];8(1):57. Available from: http://www.ncbi.nlm.nih.gov/pubmed/23497676

17. Sharp L, Finnilä K, Johansson H, Abrahamsson M, Hatschek T, Bergenmar M. No differences between Calendula cream and aqueous cream in the prevention of acute radiation skin reactions - Results from a randomised blinded trial. Eur J Oncol Nurs [Internet]. 2013 Aug [cited 2017 Mar 8];17(4):429-35. Available from: http://www.ncbi.nlm.nih.gov/pubmed/23245940

18. Pinnix C, Perkins GH, Strom EA, Tereffe W, Woodward W, Oh JL, et al. Topical Hyaluronic Acid vs. Standard of Care for the Prevention of Radiation Dermatitis After Adjuvant Radiotherapy for Breast Cancer: Single-Blind Randomized Phase III Clinical Trial. Int J Radiat Oncol. 2012 Jul;83(4):1089-94.

19. Hemati S, Asnaashari O, Sarvizadeh M, Motlagh BN, Akbari M, Tajvidi M, et al. Topical silver sulfadiazine for the prevention of acute dermatitis during irradiation for breast cancer. Support Care Cancer. 2012 Aug;20(8):1613-8.

20. Togni S, Maramaldi G, Bonetta A, Giacomelli L, Di Pierro F. Clinical evaluation of safety and efficacy of Boswellia-based cream for prevention of adjuvant radiotherapy skin damage in mammary carcinoma: a randomized placebo controlled trial. Eur Rev Med Pharmacol Sci [Internet]. 2015 Apr [cited 2016 Nov 12];19(8):1338-44. Available from: http://www.ncbi.nlm.nih.gov/pubmed/25967706

21. Rollmann DC, Novotny PJ, Petersen IA, Garces YI, Bauer HJ, Yan ES, et al. Double-Blind, Placebo-Controlled Pilot Study of Processed Ultra Emu Oil Versus Placebo in the Prevention of Radiation Dermatitis. Int J Radiat Oncol. $2015 \mathrm{Jul} ; 92(3): 650-8$.

22. Eda K, Uzer K, Murat T, Cenk U. The effects of enteral glutamine on radiotherapy induced dermatitis in breast cancer. Clin Nutr. 2016 Apr;35(2):4369.

23. Hoopfer D, Holloway C, Gabos Z, Alidrisi M, Chafe S, Krause B, et al. ThreeArm Randomized Phase III Trial: Quality Aloe and Placebo Cream Versus Powder as Skin Treatment During Breast Cancer Radiation Therapy. Clin Breast Cancer. 2015 Jun;15(3):181-190.e4.

24. Hindley A, Zain Z, Wood L, Whitehead A, Sanneh A, Barber D, et al. Mometasone Furoate Cream Reduces Acute Radiation Dermatitis in Patients Receiving Breast Radiation Therapy: Results of a Randomized Trial. Int J Radiat Oncol. 2014 Nov;90(4):748-55.

25. Lewis L, Carson S, Bydder S, Athifa M, Williams AM, Bremner A. Evaluating 
the Effects of Aluminum-Containing and Non-Aluminum Containing Deodorants on Axillary Skin Toxicity During Radiation Therapy for Breast Cancer: A 3Armed Randomized Controlled Trial. Int J Radiat Oncol. 2014 Nov;90(4):76571.

26. Chan RJ, Mann J, Tripcony L, Keller J, Cheuk R, Blades R, et al. Natural OilBased Emulsion Containing Allantoin Versus Aqueous Cream for Managing Radiation-Induced Skin Reactions in Patients With Cancer: A Phase 3, DoubleBlind, Randomized, Controlled Trial. Int J Radiat Oncol [Internet]. 2014 Nov 15 [cited 2017 Mar 8];90(4):756-64. Available from:

http://www.ncbi.nlm.nih.gov/pubmed/25151541

27. Laffin N, Smyth W, Heyer E, Fasugba O, Abernethy G, Gardner A. Effectiveness and Acceptability of a Moisturizing Cream and a Barrier Cream During Radiation Therapy for Breast Cancer in the Tropics. Cancer Nurs. 2015;38(3):205-14.

28. Alarcón B, Guarín N, Muñoz-Galindo I, Díaz J, Arévalo H. Cancer prevalence in a health insurance organization in Colombia, 2013. Rev Colomb Cancerol [Internet]. 2015 [cited 2018 Jun 11];19(4):210-21. Available from: http://www.scielo.org.co/scielo.php?script=sci_arttext\&pid=S012390152015000400004

29. Senkus E, Kyriakides S, Penault-Llorca F, Poortmans P, Thompson A, Zackrisson S, et al. Primary breast cancer: ESMO Clinical Practice Guidelines for diagnosis, treatment and follow-up. Ann Oncol [Internet]. 2013 Oct 1 [cited 2018 Jun 11];24(suppl 6):vi7-23. Available from: https://academic.oup.com/annonc/article-lookup/doi/10.1093/annonc/mdt284

30. Sociedad Española de Oncología. C, SPARC (Organization). Oncología. Vol. 28, Oncología (Barcelona). Ediciones Cutor; 2005. 24-29 p.

31. Eficacia de la administración enteral de glutamina en enfermos quemados graves. Med Intensiva [Internet]. 2005 Feb 1 [cited 2020 Jul 8];29(1):72-4. Available from: https://linkinghub.elsevier.com/retrieve/pii/S0210569105742030

32. Peng X, Yan H, You Z, Wang P, Wang S, Taylor PM. Clinical and protein metabolic efficacy of glutamine granules-supplemented enteral nutrition in severely burned patients. Burns. 2005 May;31(3):342-6.

33. Gosselin, Tracy K; Schneider, Susan M; Plambeck, Mary Ann; Rowe K. A Prospective Randomized, Placebo-Controlled Skin Care Study in Women Diagnosed With Breast Cancer Undergoing Radiation Therapy. Oncol Nurs Forum. 2010;37(5):619-26.

34. Meuleneire F. A vapour-permeable film dressing used on superficial wounds. $\mathrm{Br}$ J Nurs [Internet]. 2014 Aug 12 [cited 2018 Jun 11];23(Sup15):S36-43. Available from: http://www.magonlinelibrary.com/doi/10.12968/bjon.2014.23.Sup15.s36

35. Diggelmann K V, Zytkovicz AE, Tuaine JM, Bennett NC, Kelly LE, Herst PM. Mepilex Lite dressings for the management of radiation-induced erythema: a systematic inpatient controlled clinical trial. Br J Radiol [Internet]. 2010 Nov 13 [cited 2018 Jun 12];83(995):971-8. Available from: http://www.birpublications.org/doi/10.1259/bjr/62011713

36. Paterson D. Randomized Intra-patient Controlled Trial of Mepilex Lite Dressings 
versus Aqueous Cream in Managing Radiation-Induced Skin Reactions Postmastectomy. J Cancer Sci Ther [Internet]. 2012 [cited 2018 Jun 12];04(11). Available from: https://www.omicsonline.org/randomized-intra-patientcontrolled-trial-of-mepilex-lite-dressings-versus-aqueous-cream-in-managingradiation-induced-skin-reactions-postmastectomy-19485956.1000166.php?aid $=8898$

37. Farhan F, Kazemian A, Alagheband H. Topical betamethasone for the prevention of acute radiation dermatitis in breast cancer patients. Iran J Radiat Res. 2003;1(2):105-11.

38. Schmuth M, Wimmer MA, Hofer S, Sztankay A, Weinlich G, Linder DM, et al. Topical corticosteroid therapy for acute radiation dermatitis: a prospective, randomized, double-blind study. Br J Dermatol. 2002 Jun;146(6):983-91.

39. Shukla PN, Gairola M, Mohanti BK, Rath GK. Prophylactic beclomethasone spray to the skin during postoperative radiotherapy of carcinoma breast: a prospective randomized study. Indian J Cancer. 43(4):180-4.

40. Boström A, Lindman H, Swartling C, Berne B, Bergh J. Potent corticosteroid cream (mometasone furoate) significantly reduces acute radiation dermatitis: results from a double-blind, randomized study. Radiother Oncol. 2001 Jun;59(3):257-65.

41. Kramer A, Dissemond J, Kim S, Willy C, Mayer D, Papke R, et al. Consensus on Wound Antisepsis: Update 2018 [Internet]. Vol. 31, Skin Pharmacology and Physiology. S. Karger AG; 2018 [cited 2020 Mar 27]. p. 28-58. Available from: http://www.ncbi.nlm.nih.gov/pubmed/29262416

42. Kirova YM, Fromantin I, De Rycke Y, Fourquet A, Morvan E, Padiglione S, et al. Can we decrease the skin reaction in breast cancer patients using hyaluronic acid during radiation therapy? Results of phase III randomised trial. Radiother Oncol [Internet]. 2011 Aug 1 [cited 2018 Jun 11];100(2):205-9. Available from: https://www.sciencedirect.com/science/article/pii/S0167814011002064?via\%3Di hub

43. Liguori V, Guillemin C, Pesce GF, Mirimanoff RO, Bernier J. Double-blind, randomized clinical study comparing hyaluronic acid cream to placebo in patients treated with radiotherapy. Radiother Oncol. 1997 Feb;42(2):155-61.

44. Richardson J, Smith JE, McIntyre M, Thomas R, Pilkington K, Kitagawa T. Aloe Vera for Preventing Radiation-induced Skin Reactions: A Systematic Literature Review. Clin Oncol. 2005 Sep;17(6):478-84.

45. Rao S, Hegde S, Baliga-Rao M, Palatty P, George T, Baliga M. An Aloe VeraBased Cosmeceutical Cream Delays and Mitigates Ionizing Radiation-Induced Dermatitis in Head and Neck Cancer Patients Undergoing Curative Radiotherapy: A Clinical Study. Medicines [Internet]. 2017 Jun 24 [cited 2018 Jun 11];4(3):44. Available from: http://www.mdpi.com/2305-6320/4/3/44

46. Villanueva Ramos T, Alcalá Pérez D, Vega Gonzales M, Peralta PEdrero ML, MEdina Bojórquez A, Barrera Cruz A. Guía de práctica clínica para prevención y tratamiento de la radiodermatitis aguda. Dermatol Rev Mex. 2012;56 (1):11.

47. Théberge V, Harel F, Dagnault A, al. et. Use of axillary deodorant and effect on acute skin toxicity during radiotherapy for breast cancer: a prospective 
randomized noninferiority trial. Int J Radiat Oncol Biol Phys. 2009 Nov;75(4):1048-52.

48. Watson LC, Gies D, Thompson E, Thomas B. Randomized control trial: evaluating aluminum-based antiperspirant use, axilla skin toxicity, and reported quality of life in women receiving external beam radiotherapy for treatment of Stage 0, I, and II breast cancer. Int J Radiat Oncol Biol Phys. 2012 May;83(1):e29-34.

49. Gee A, Moffitt D, Churn M, Errington RD. A randomised controlled trial to test a non-metallic deodorant used during a course of radiotherapy. J Radiother Pract. 2000 Dec;1(04):205-12.

50. Pardo Masferrer J, Murcia Mejía M, Vidal Fernández M, Alvarado Astudillo A, Hernández Armenteros ML, Macías Hernández V, et al. Prophylaxis with a cream containing urea reduces the incidence and severity of radio-induced dermatitis. Clin Transl Oncol [Internet]. 2010 Jan 30 [cited 2018 Jun 12];12(1):43-8. Available from: http://link.springer.com/10.1007/s12094-010$0465-0$

51. Becker-Schiebe M, Mengs U, Schaefer M, Bulitta M, Hoffmann W. Topical Use of a Silymarin-Based Preparation to Prevent Radiodermatitis. Strahlentherapie und Onkol [Internet]. 2011 Aug 22 [cited 2018 Jun 12];187(8):485-91. Available from: http://link.springer.com/10.1007/s00066-011-2204-z 